\title{
Spatiotemporal Optical Vortices
}

\author{
N. Jhajj, ${ }^{*}$ I. Larkin, E. W. Rosenthal, S. Zahedpour, J. K. Wahlstrand, and H. M. Milchberg ${ }^{\dagger}$ \\ Institute for Research in Electronics and Applied Physics, University of Maryland, \\ College Park, Maryland 20742, USA
}

(Received 17 May 2016; revised manuscript received 30 June 2016; published 9 September 2016; corrected 20 December 2017)

\begin{abstract}
We present the first experimental evidence, supported by theory and simulation, of spatiotemporal optical vortices (STOVs). A STOV is an optical vortex with phase and energy circulation in $a$ spatiotemporal plane. Depending on the sign of the material dispersion, the local electromagnetic energy flow is saddle or spiral about the STOV. STOVs are a fundamental element of the nonlinear collapse and subsequent propagation of short optical pulses in material media, and conserve topological charge, constraining their birth, evolution, and annihilation. We measure a self-generated STOV consisting of a ring-shaped null in the electromagnetic field about which the phase is spiral, forming a dynamic torus that is concentric with and tracks the propagating pulse. Our results, here obtained for optical pulse collapse and filamentation in air, are generalizable to a broad class of nonlinearly propagating waves.
\end{abstract}

DOI: 10.1103/PhysRevX.6.031037

\section{INTRODUCTION}

Vortices-localized regions in which the flow of some quantity such as mass or electromagnetic energy circulates about a local axis - are a common and fundamental element of classical [1] and quantum fluids [2-4] as well as optics [5]. In quantum fluids, the circulating quantity is the spatial atomic probability density; in optics, it is the electromagnetic energy density. Both densities are expressed as the magnitude squared of a complex scalar field $\psi=u e^{i \Phi}$ derivable from a Schrödinger-like equation (SE), where the vortex circulation is $\Gamma=\oint_{c} \nabla \Phi \cdot d \mathbf{l}, u$ and $\Phi$ are real scalar fields, and the integral is on a closed contour about the local axis. A nonzero value of $\Gamma$ implies a discontinuity or "defect" in $\Phi$. Furthermore, the single valuedness of fields demands that the circulation be quantized, $\Gamma=2 m \pi$, where $m$, an integer, is called the topological charge. Because $\Gamma$ remains constant as one shrinks the contour around the local axis, or vortex core, the phase $\Phi$ becomes undefined at the core center (phase singularity), where the field magnitude is necessarily zero.

In optics, vortices have been heavily investigated for decades, but as far as we know, the types that have been studied are entirely those that can be supported by

\footnotetext{
*Corresponding author. njhajj@gmail.com

Corresponding author. milch@umd.edu

Published by the American Physical Society under the terms of the Creative Commons Attribution 3.0 License. Further distribution of this work must maintain attribution to the author $(s)$ and the published article's title, journal citation, and DOI.
}

Subject Areas: Interdisciplinary Physics, Optics, Photonics monochromatic waves; that is, they have phase and energy circulation purely in the spatial domain.

Here, we present experimental evidence, backed by theory and simulation, for a new type of dynamic phase vortex that mediates energy flow spatially and temporally. The spatiotemporal character of the vortex dictates that energy flow near the vortex core is saddle or spiral depending on the sign of dispersion of the propagation medium. Further, we show that vortex formation is an inherent feature of nonlinear collapse arrest, and that vortex dynamics are associated with changes to the morphology of a nonlinearly propagating pulse.

In an early, influential publication, Nye and Berry introduced the concept of dislocations (field nulls) to wave theory and gave many examples [6]. A well-known example of a vortex in linear optics is the linearly polarized Laguerre Gaussian (LG) mode $E_{p l}(r, \varphi, z)$ of integer radial index $p \geq 0$ and nonzero integer azimuthal index $l$, with azimuthal dependence $\exp (i l \varphi)$. This mode has an on-axis field null, a topological charge $l=\Gamma / 2 \pi$, an orbital angular momentum (OAM) of $N l \hbar$, where $N$ is the number of photons in the beam, and a spiral energy density flux about the propagation axis [7]. The phase singularity at beam center is called a screw dislocation [6]. Such modes can be generated by phase plates [8]. LG modes are solutions to the paraxial wave equation $i \partial \psi / \partial z+\nabla_{\perp}^{2} \psi=0$ for propagation along $z$, which is of the SE form with zero potential [7]. An example of a LG field at $z=0$ with a null on axis is $E_{0, \pm 1}\left(\mathbf{r}_{\perp}\right) \propto[x \pm i y] w_{r}^{-1}\left\{e^{-\left|\mathbf{r}_{\perp}\right|^{2} / w_{r}^{2}}\right\}$, where $\mathbf{r}_{\perp}=x \hat{\mathbf{x}}+y \hat{\mathbf{y}}$ and $w_{r}$ is the beam waist.

Now consider, as an example, a construction similar mathematically to the LG field but physically quite different: the pulsed Gaussian field $E\left(\mathbf{r}_{\perp}, \xi\right) \propto\left[\xi / w_{\xi} \pm i x / w_{r}\right] \times$ $\left\{e^{-\left|\mathbf{r}_{\perp}\right|^{2} / w_{r}^{2}} e^{-\xi^{2} / w_{\xi}^{2}}\right\}$, where $\xi=v_{g} t-z$ is the local position 
in a frame moving with the pulse group velocity $v_{g}$, and $w_{\xi}$ is the axial pulse length. This field has a moving null at $(x=0, \xi=0)$ along the $y$ axis, perpendicular to the direction of propagation, and the circulation $\Gamma$ on closed spatiotemporal contours in $(x, \xi)$ around the null is quantized. We refer to this apparently previously unexplored object as a spatiotemporal optical vortex (STOV). We contrast the STOV construction with other recent work, such as by Eilenberger et al. [9] or Mihalache et al. [10], where beams with nontrivial spatiotemporal coupling are created with embedded vorticity, but the vorticity is fundamentally similar to the LG construction given above, where phase and energy circulation from the vortex occur only in the transverse spatial dimensions. We refer to optical vortices with phase circulation solely in spatial dimensions as spatial optical vortices, and note that, as far as we know, this definition encompasses all optical vortices studied to date. Outside of optics there has been theoretical and experimental work on systems with analogs of the STOV construction, such as work by Nicholls and Nye in acoustics [11] and Kartashov et al. in quantum fluids [12].

In this paper, we demonstrate that STOVs are a fundamental and universal feature of optical pulse collapse and arrest in self-focusing media. Their existence in nonlinear ultrafast pulse propagation appears to be ubiquitous, and their creation, motion, and destruction is strongly linked to the complex spatiotemporal evolution of the pulse.

\section{SPATIOTEMPORAL OPTICAL VORTICES}

Optical collapse is a fundamental phenomenon in nonlinear optics [13]. It occurs when the laser-pulse-induced change in the medium's refractive index generates a self-lens whose focusing strength increases with intensity. Above a critical power level $\left(P_{\mathrm{cr}}\right)$, self-lensing exceeds diffraction, and the pulse experiences runaway self-focusing. In the absence of "arrest" mechanisms terminating self-focusing, the pulse would collapse to a singularity. In reality, additional physical effects intervene. For example, in the case of femtosecond filamentation in ionizing media [14], plasma generation acts to defocus the pulse when the peak selffocused beam intensity reaches the ionization threshold. Other collapse arrest mechanisms include dispersioninduced pulse lengthening [15], cascaded third-order nonlinearities [16], vectorial effects from beam nonparaxiality [17], and, in the case of relativistic self-focusing, electron cavitation [18].

In both calculations and simulations [19,20], it has been found that the following modified paraxial equation for wave evolution, in the form of a time-dependent nonlinear Schrödinger equation, is well suited to describe optical pulse collapse and collapse arrest for beam propagation along $z$ :
$2 \frac{\partial}{\partial z}\left(i k-\frac{\partial}{\partial \xi}\right) \psi+\nabla_{\perp}^{2} \psi-\beta_{2} \frac{\partial^{2} \psi}{\partial \xi^{2}}+k^{2} V\{\psi\}=0$.

Here, $\quad E=\psi\left(\mathbf{r}_{\perp}, z, \xi\right) e^{i(k z-\omega t)}$ is the dimensionless electric field component, $\psi$ is the pulse envelope, $\beta_{2}=c^{2} k_{0}\left(\partial^{2} k / \partial \omega^{2}\right)_{0}$ is the dimensionless group velocity dispersion at $\omega=\omega_{0}, \mathbf{r}_{\perp}$ and $\xi$ are as before, and $z$ is the lab frame axial position of the pulse. The physics of self-focusing and arrest is contained in the functional $V\{\psi\}$. To demonstrate toroidal STOV generation in the arrest of self-focusing collapse, we perform propagation simulations using Eq. (1), imposing azimuthal symmetry, and include electronic, rotational, and ionization nonlinearities in $V\{\psi\}$ [19,21]. The Gaussian input pulse is $3 \mathrm{~mJ}, 45 \mathrm{fs}$ (Gaussian FWHM) with an input waist $w_{0}=1 \mathrm{~mm}$, and the propagation medium is atmospheric pressure air. As our experiments (see Sec. III) are intentionally operated in the single filamentation regime, where multifilamentary modulational instabilities are precluded, azimuthal symmetry is a good approximation [14]. Figure 1(a) is a postcollapse plot of the pulse phase at $z=120 \mathrm{~cm}$, which shows the emergence of two oppositely wound and oppositely propagating STOVs $\left[v_{1}(+1\right.$ charge, forward) and $v_{2}$ ( -1 charge, backward)] entrained between the higher intensity core of the beam and the beam periphery. The delayed rotational nonlinearity from the $\mathrm{N}_{2}$ and $\mathrm{O}_{2}$ air constituents [22] forms an additional vortex pair $\sim 100 \mathrm{fs}$ behind the main pulse, $v_{3}\left(+1\right.$, forward) and $v_{4}(-1$, backward), where $v_{3}$ is shown bisected in Fig. 1(a) and $v_{4}$ has exited the simulation window. Further evolution of the simulation shows that $v_{2}$ and $v_{3}$ collide and annihilate, while $v_{1}$ continues propagating with the most intense part of the pulse (which we discuss later). We note that the delayed generation of $v_{3}$ and $v_{4}$, where the pulse intensity is many orders of magnitude weaker than at its peak, shows that STOVs can also be generated linearly by an imposed spatiotemporal index transient. STOVs are not merely mathematical free-riders on intense propagating pulses: as we show, a real energy flux $\mathbf{j}$ circulates either as a saddle (for $\beta_{2}>0$ ) or as a spiral (for $\beta_{2}<0$ ) in the $\left(\mathbf{r}_{\perp}, \xi\right)$ plane surrounding the STOV core.

To understand the generation of these spatiotemporal toroidal structures, recall that phase vortices in fields are closely associated with localized field nulls [6]. In arrested self-focusing, field nulls occur as a natural part of the dynamics and spawn toroidal vortices of opposite charge. This can be illustrated by the toy model of Fig. 2, which shows the effect on a beam of abruptly spatially varying self-induced change in refractive index, which we model here as a sharp transverse step. We consider left-to-right propagation of the "half plane wave" pulse $E(x, z, \xi)=$ $E_{0}[\delta+h(x)] e^{-\left(\xi / \xi_{0}\right)^{2}} e^{i\left[z\left(k-\omega / v_{g}\right)-\omega \xi / v_{g}\right]} e^{i \phi_{\mathrm{NL}}(x, z, \xi)}$, neglecting dispersion and diffraction, where $\xi$ and $z$ are as before, $\delta \ll 1, h(x)=1$ for $x<0$ and 0 otherwise, $\phi_{\mathrm{NL}}(x, z, \xi)=$ $k n_{2}|E(x, z, \xi)|^{2} z$ is the sharply stepped nonlinear Kerr phase, and $n_{2}$ is the nonlinear index of refraction. At 


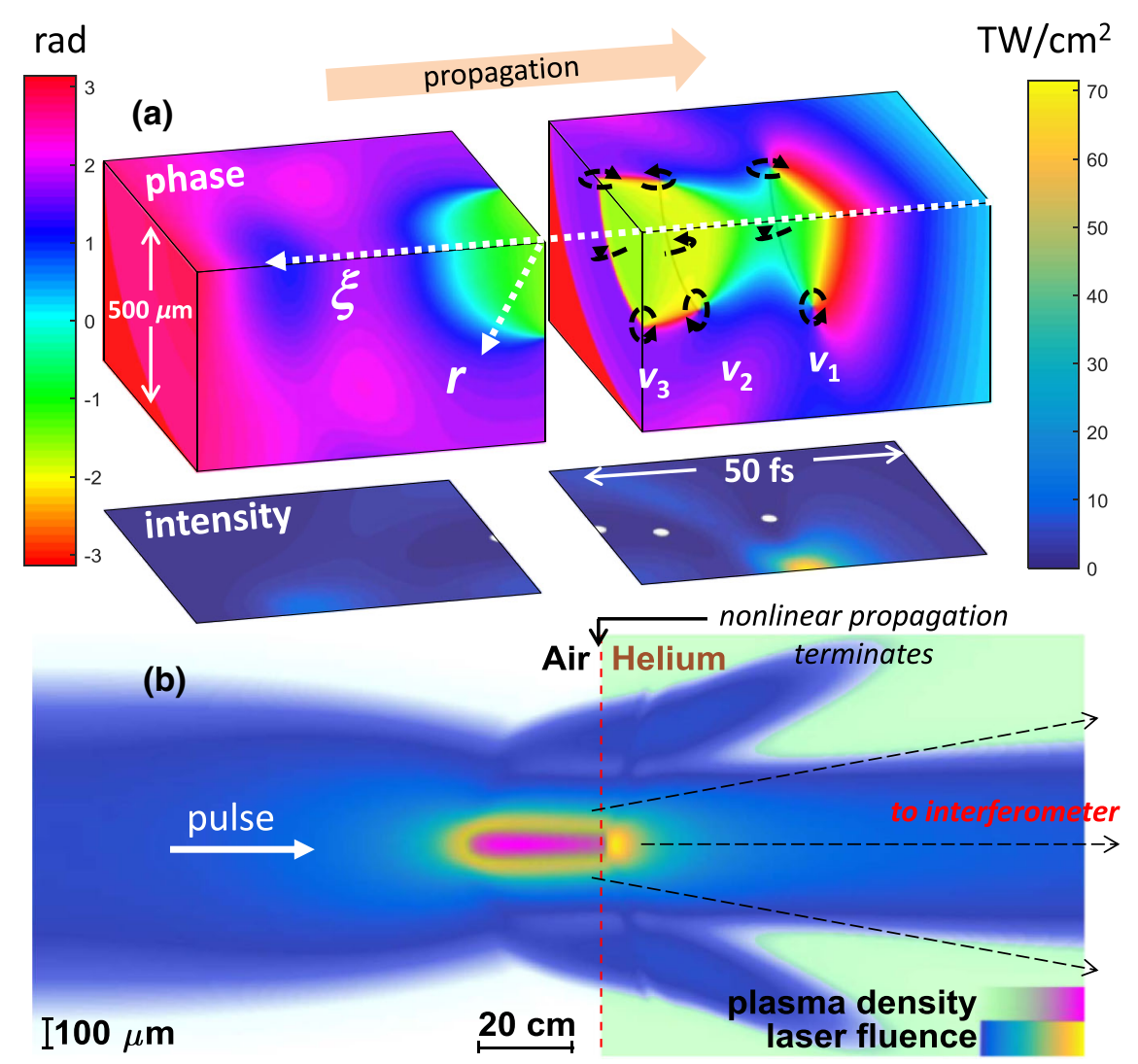

FIG. 1. (a) Phase and intensity projections of simulated pulse, in local coordinates, showing STOV generation. Propagation medium: atmospheric pressure air. Black dashed lines encircle vortices $v_{1}, v_{2}$, and $v_{3}$, and arrows point in the direction of increasing phase. Our phase winding convention considers a $\left(\xi-\xi_{0}\right)+i\left(r-r_{0}\right)$ winding about a null at $\left(\xi_{0}, r_{0}\right)$ to denote a +1 STOV, giving the $v_{1}$ STOV a +1 charge. The white dots in the intensity projection are centered on the locations of vortices $v_{1}, v_{2}$, and $v_{3}$. (b) Simulation of an air filament crossing the air-helium boundary for the conditions of (a), showing the beam fluence and plasma density. Nonlinear propagation terminates as the beam transitions from air to helium, whereupon the beam and a reference pulse (not shown; see Appendix A) are directed to an interferometer.

$z=0$, the phase fronts are aligned for all $x$. As the pulse propagates, the Gaussian intensity distribution causes the front of the pulse to redshift and the back of the pulse to blueshift, with spatiotemporal phase front shear developing between the pulse "core" at $x<0$ and periphery at $x>0$. When the propagation reaches $z=z_{v}=\pi\left(k n_{2} E_{0}^{2}\right)^{-1}$, the peak of the pulse in the core is $\pi$ out of phase with the periphery, and the electric field magnitude nulls out at the single point $\left(\xi=0, x=0, z=z_{v}\right)$, marked by a circle, forming an "edge dislocation" [6]. Upon further propagation, continued phase shearing spawns two null points of opposing $( \pm 1)$ phase winding, marked by circles $\left(z=2 z_{v}\right)$. These two STOVs, whose axes are along $y$ (perpendicular to page), immediately begin moving apart: one vortex advances in time towards the front of the pulse while the other vortex moves to the back. Similar dynamics are theorized to exist in monochromatic breather solitons, where ring-shaped vortices are formed quasiperiodically throughout propagation [23].

These general features occur in simulations of self-focusing collapse arrest. In Fig. 3, we show 2D profiles of the pulse's phase $\phi\left(r, z, \xi=\xi_{v}\right)$ and intensity $c\left|E\left(r, z, \xi=\xi_{v}\right)\right|^{2} / 8 \pi$ for a simulation using Eq. (1), tracking the moving plane $\xi=\xi_{v}$, where the phase singularity and field null first appear. Log lineouts of the intensity (normalized to $10^{13} \mathrm{~W} / \mathrm{cm}^{2}$ ) are overlaid on the 2D profiles. As the initial $(z=0)$ Gaussian input beam self-focuses, a strongly peaked high-intensity central core (similar to the Townes profile [24]) develops, surrounded by a lower-intensity periphery, with a sharp transition knee between them $(z=156 \mathrm{~cm})$. The associated phase plot shows the central core having accumulated a much larger nonlinear phase shift than the periphery. During collapse, the knee moves radially inward, preventing phase shear from accumulating substantially at any particular radial location. However, as the core peak intensity rises to the point where ionization begins $(z=160 \mathrm{~cm}$, not shown), the location of the intensity knee stabilizes and highly localized phase shear builds up, greatly steepening the transition between core and periphery, with the field beginning to dip towards a null $(z=166 \mathrm{~cm})$. Finally, 


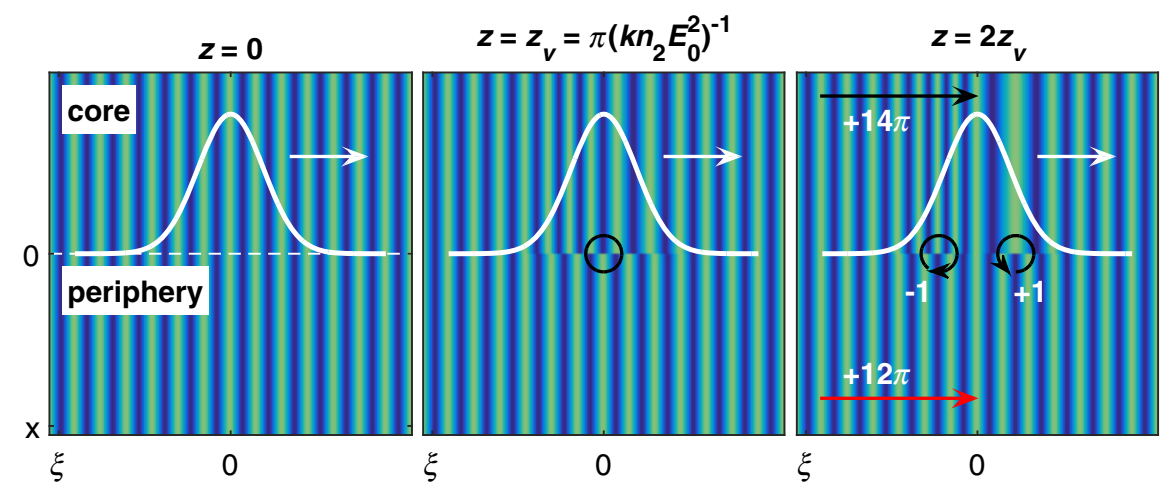

FIG. 2. Toy model showing birth of a vortex pair via spatiotemporal phase front shear. The white curve and arrow depicts the axial (temporal) intensity profile and propagation direction, while the "core" and "periphery" labels denote the spatial intensity step. The $z=0$ panel shows the initial condition where phases are aligned, the $z=z_{v}$ panel shows the birth of the null (vortices overlap), and the $z=2 z_{v}$ panel shows continued shear carrying the vortices apart, with the +1 vortex moving to the temporal front and the -1 vortex moving backward. Our phase winding convention considers a $\left(\xi-\xi_{0}\right)+i\left(x-x_{0}\right)$ winding about a null at $\left(\xi_{0}, x_{0}\right)$ to denote a +1 STOV. In the third panel, red and black arrows indicate the direction of increasing phase. The two arrows connect the same lines of constant phase; the arrows show that the phase difference between head and tail is ill defined due to the vorticity of the -1 STOV.

only a short propagation distance later $(z=167 \mathrm{~cm})$, sufficient shear develops that the core and periphery are $\pi$ out of phase at the slice $\xi=\xi_{v}$, with the dip in the simulation becoming orders of magnitude deeper, forming a ring-shaped null surrounding a core of relatively flat intensity and phase. Note that the core-periphery phase difference $\Delta \phi_{\mathrm{cp}}$ jumps by $2 \pi$ between $z=166$ and $167 \mathrm{~cm}$. [As seen in the computation of Fig 1(a), the null then spawns two oppositely charged $( \pm 1)$ toroidal vortices that propagate forward and backward.] The plane $\xi=\xi_{v}$ is still shown at $z=178 \mathrm{~cm}$, but the vortices have now migrated out of that plane. Later in the paper, we derive and discuss equations of motion describing vortex dynamics within the moving reference frame.
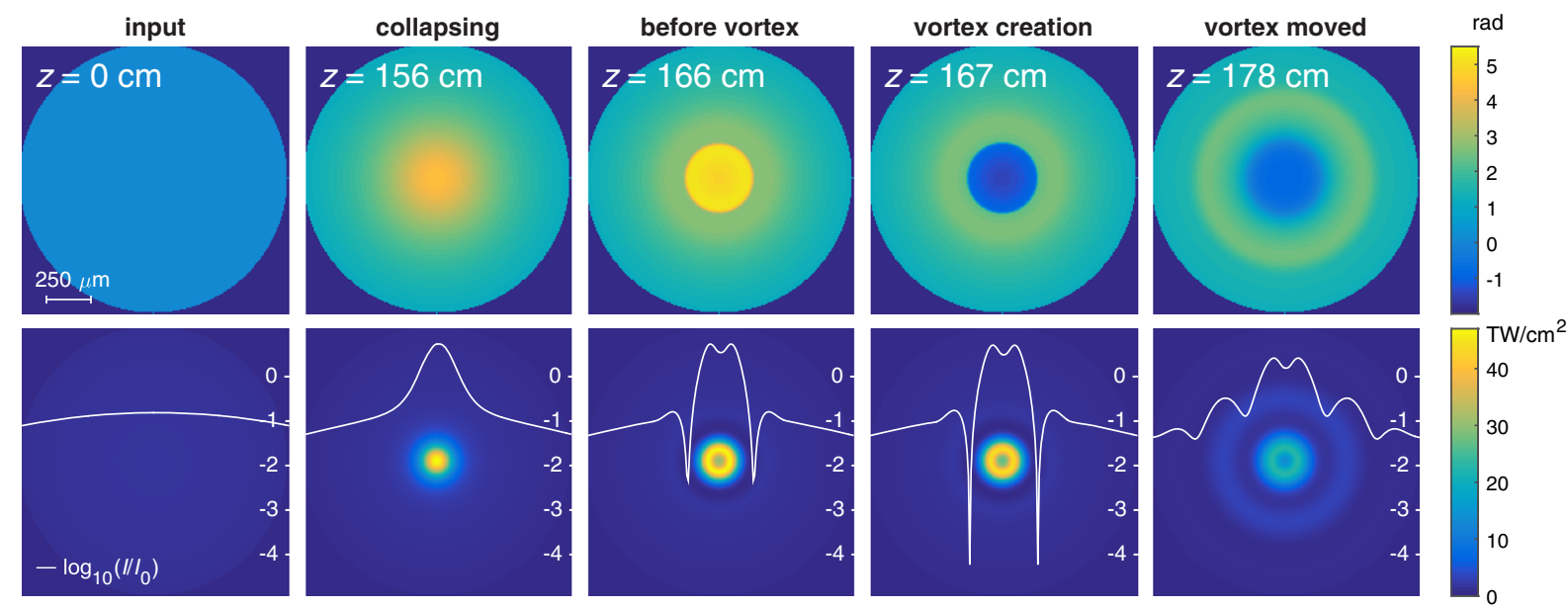

FIG. 3. Simulations of beam phase (top) and intensity (bottom) at the axial or temporal slice $\xi_{v}$, where the STOV pair first appears. From left to right, the pulse is advancing along $z$, with the input shown at $z=0$, a collapsing beam at $z=156 \mathrm{~cm}$, ionization onset at $160 \mathrm{~cm}$ (not shown), just before the vortices spawn at $166 \mathrm{~cm}$, just after the vortices spawn at $167 \mathrm{~cm}$, and an image where the vortex pair has moved out of the $\xi=\xi_{v}$ plane. The linear intensity images are overlaid with centered lineouts $\log _{10}\left(I / I_{0}\right)$, where $I_{0}=10^{13} \mathrm{~W} / \mathrm{cm}^{2}$. Experimental parameters are used as code inputs: $w_{0}=1.3 \mathrm{~mm}$, pulse energy $2.8 \mathrm{~mJ}\left(P / P_{\mathrm{cr}}=6.4\right)$, pulse FWHM 45 fs. 
frequency. This leads to the vortex appearing in the spatiospectral as well as the spatiotemporal domain. In Appendix A 1, we discuss a Gaussian beam with a temporally centered toroidal STOV and show that the spatiospectral representation of the beam possesses a vortex with the same spatial radius. We note that the relationship between vorticity in the spatiotemporal and spatiospectral domains is, in general, complex, with the full field distribution (including vortices) in one domain contributing to an individual vortex in the conjugate domain. Because our experimental images identify STOVs after optical collapse arrest has occurred, our results avoid this complexity, as simulations show that by this point there is only one $( \pm 1)$ STOV propagating with the filamenting pulse.

Direct measurement of the spatial phase and intensity profile of a filament in midflight is not amenable to standard techniques; the use of relay imaging or beam splitters is subject to the severe distorting effects of nonlinear propagation and material damage. However, by interrupting nonlinear beam propagation by an air-helium interface, the in-flight beam intensity and phase profile can be linearly imaged through helium, taking advantage of the very large difference in instantaneous nonlinear response between helium and $\operatorname{air}\left(n_{2, \mathrm{He}} / n_{2, \text { air }} \approx 0.04[22,25]\right)$. This helium cell technique was first employed by Ting et al. [26] to measure the in-flight intensity profile of a femtosecond filament. Here, we extend the technique to also enable measurement of the pulse transverse phase profile. In Appendix A, we discuss the details of how we produce the air-helium interface and how the <4-mm-thick air-helium transition layer is sufficiently thin to enable distortion-free imaging of the air filament cross section. The interface is movable along the laser propagation axis to allow midflight filament intensity and phase imaging over the full propagation path.

A conceptual view of the experiment is presented in Fig. 1(b), where we show the postcollapse pulse from the simulation of Fig. 1(a) encountering the air-helium interface and terminating nonlinear propagation, after which it is relay imaged through a folded wave front interferometer and combined there with a weak femtosecond reference beam with flat spatial and spectral phase. The resulting interferogram encodes the 2D spatial phase and intensity of the in-flight filamenting (signal) beam at the air-helium interface, averaged over the $\sim 20-\mathrm{nm}$ bandwidth of the $\lambda=800 \mathrm{~nm}$ reference arm. In effect, the reference arm spectrally gates the STOV, resulting in a spatiospectral interferogram centered at the reference pulse bandwidth. Spectral gating is crucial to our measurements. If the reference arm had the same wide bandwidth as nonlinearly generated in the signal (filamenting) arm, the signature of the STOV, which is present over a smaller spectral window, would be washed out. A detailed discussion of the experimental setup, the spatiospectral representation of STOVs, and the interferometric analysis is found in Appendix A.

\section{B. Experimental results}

The experiment is performed by scanning the helium cell axial position over a range covering both precollapse and postcollapse propagation for all energies used. The data consist of a densely spaced collection of beam intensity and phase images at various cell positions $\left(z_{h}\right)$ and input pulse energies $\left(\varepsilon_{i}\right)$. For all measurements, collimated beams are launched with $w_{0}=1.3 \mathrm{~mm}$ and FWHM intensity pulse width $\tau=45$ fs.

Figure 4 shows the beam on-axis phase shift $\Delta \phi$ with respect to the interferometric reference pulse as a function of $P / P_{\text {cr }}$ at a fixed position of $z_{h}=150 \mathrm{~cm}$ after launch, where $P_{\mathrm{cr}}=3.77 \lambda^{2} / 8 \pi n_{0} n_{2}$ for our Gaussian input beam profile and $P=\epsilon_{i} / \tau$ is the input power. The red points are averages over 2600 shots (blue points) in 150 energy bins. It is important to note that the scatter in $\Delta \phi$ of roughly $\pm 1 \mathrm{rad}$ at any given laser power is constant across all powers measured, including $P / P_{\text {cr }} \ll 1$, where we could not detect any nonlinear phase. Therefore, the scatter is due to the shot-to-shot interferometric instability of the measurement and is not intrinsic to the filamentation process. The most striking aspect of the plot is the abrupt jump in beam central phase of approximately $\sim 2 \pi$ at $P / P_{\text {cr }} \sim 5$. The phase goes from positive and rapidly increasing (increasing self-focusing) to abruptly negative (defocusing), providing a clear signature of the transition from the precollapse to the postcollapse beam. For nominally constant laser power right at the jump, the phase fluctuates in the range $\sim \pm \pi$, showing the extreme sensitivity of the phase flip.

A more revealing way to display what is happening at the collapse is shown in Fig. 5. Here, for given $z_{h}$, the phase images $\left(z_{h}, \varepsilon_{i}\right)$ are searched for $\varepsilon_{i}$ or $P / P_{\mathrm{cr}}$, where the

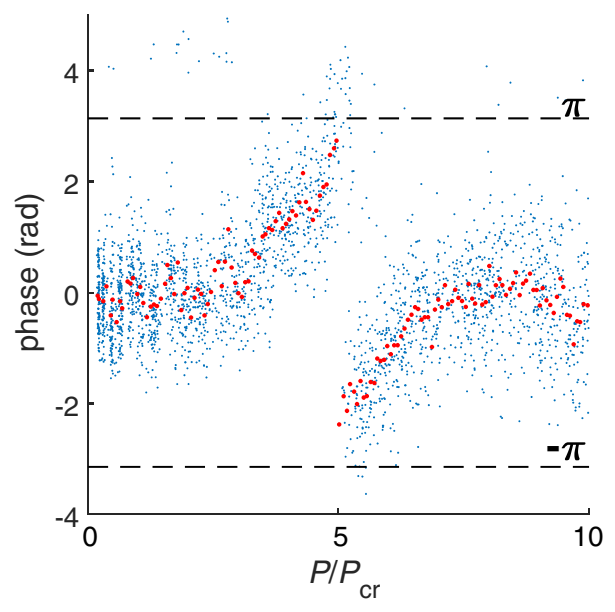

FIG. 4. Beam on-axis phase shift (with respect to flat-phase reference arm) as a function of pulse power at $z_{h}=150 \mathrm{~cm}$. The phase jumps abruptly by $\sim 2 \pi$ at $P / P_{\text {cr }} \sim 5$, providing a clear signature of the transition from the precollapse to the postcollapse beam. The red points are averages over 2600 shots (blue points) in 150 energy bins. 


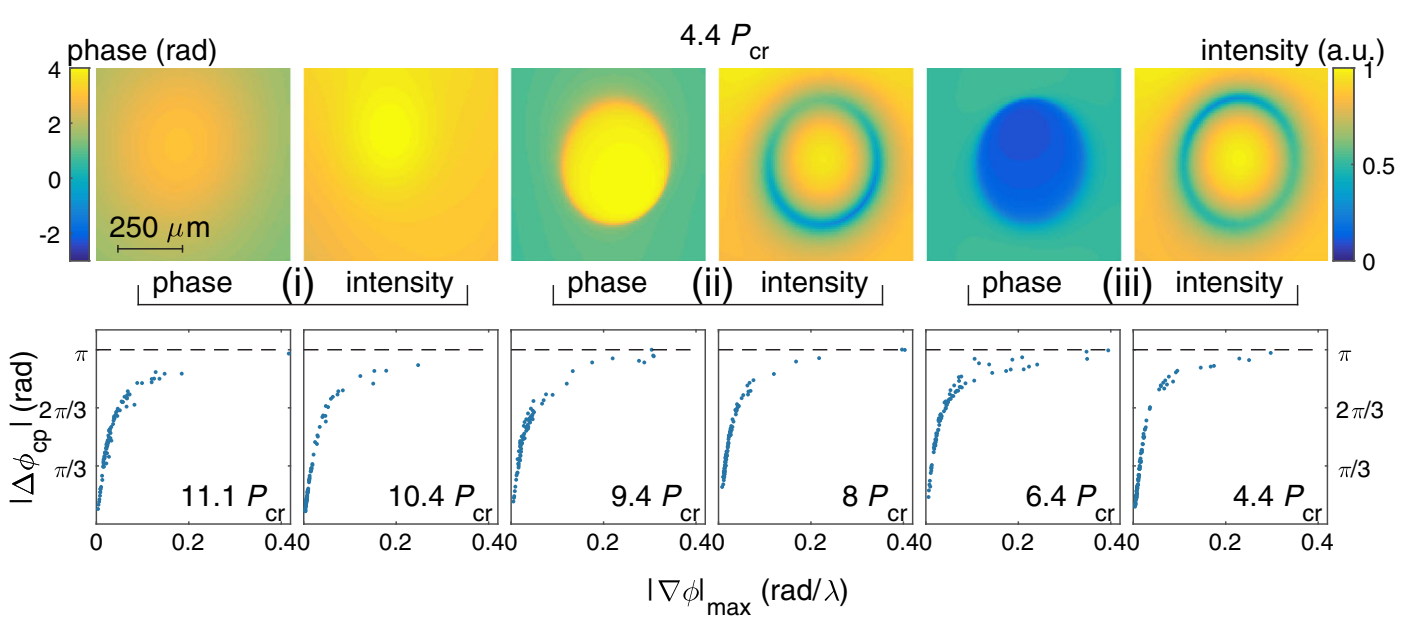

FIG. 5. Top row: Retrieved spatial phase (radians) and intensity (arbitrary units) images at $z=165 \mathrm{~cm}, P / P_{\text {cr }}=4.4$ for (i) a precollapse beam and (ii) and (iii) beams where a vortex ring is on either side of the reference central wavelength of $800 \mathrm{~nm}$. The bottom row shows that as the maximal phase gradient in the images increases, the maximal phase shift saturates at $\pi$ for all cases of $P / P_{\text {cr }}$ leading to beam collapse.

central phase appears to randomly flip sign from shot to shot. These are the powers at which pulse collapse is observed for each position, just as $P / P_{\mathrm{cr}} \sim 5$ is for $z_{h}=$ $150 \mathrm{~cm}$ in Fig. 4. The top row of Fig. 5 shows beam phase and intensity images for input power $P / P_{\text {cr }}=4.4$ (at $\left.z_{h}=165 \mathrm{~cm}\right)$. Because the onset of collapse arrest is extremely sensitive to fluctuations in the beam energy (as seen in Fig. 4), these images span the possibilities of prearrest through postarrest of the collapse, and typically three types of images appear. Figure 5(i) shows strongly peaked intensity and positive phase; the beam is collapsing, but arrest has not yet begun. Figure 5(ii) shows radically different images, as does Fig. 5(iii): the intensity images show narrow ring-shaped nulls embedded in a relatively smooth background, and the phase images show a sharp yet smoothly transitioning jump close to $\pi$ or $-\pi$ across the rings, with the phase jumps flipped between Figs. 5(ii) and 5(iii). We note that the smooth phase transition from the periphery to the core rules out $2 \pi$ phase ambiguities in interferometric phase extraction.

The bottom row of Fig. 5 plots, for a range of $P / P_{\mathrm{cr}}$, the phase difference $\left|\Delta \phi_{\mathrm{cp}}\right|$ between the core and periphery of the beam. To do this, for each phase image we compute the difference between the maximum and minimum values of the phase within a $60-\mu \mathrm{m}$ box centered about the largest spatial phase gradient, the radial location of which defines core and periphery. For each nominal value of $P / P_{\text {cr }}$, it is clear that as the phase gradient becomes large, the phase difference saturates at $\pi$. Near saturation, roughly $50 \%$ of the shots have the core phase advanced from the periphery while the others show the reverse.

The evidence from Figs. 4 and 5, and comparison to the simulation of Fig. 3, strongly suggests that we are imaging spatiotemporal vortex rings: the abrupt appearance of ringshaped nulls in the field magnitude accompanied by $2 \pi$ phase jumps in $\left|\Delta \phi_{\mathrm{cp}}\right|$ across the null-these are exactly the signatures of a vortex. Because the circulation around a general singly charged vortex is $2 \pi$, examining our vortex in the spatiospectral domain $\left(\mathbf{r}_{\perp}, \omega_{\xi}\right)$, one would expect, depending on the sign of vortex winding, that the coreperiphery phase difference $\left|\Delta \phi_{\mathrm{cp}}\right|$ jumps by $2 \pi$ from $\omega_{\xi}$ slices just before the vortex $\left(\Delta \phi_{\mathrm{cp}}= \pm \pi\right)$ to $\omega_{\xi}$ slices just after $\left(\Delta \phi_{\mathrm{cp}}=\mp \pi\right)$. For example, before a vortex of charge +1 , the core is phase advanced with respect to the periphery; after the vortex, it is phase retarded. This is exactly what we observe experimentally and what is predicted in the simulation of Fig. 3 and its spatiospectral counterpart, where even the $\sim 400-\mu \mathrm{m}$ diameter of the vortex ring is accurately determined. Of the four STOVs that simulations show are generated at collapse arrest in air [see discussion of Fig. 1(a)], only the temporally foremost +1 STOV does not annihilate or separate from the bulk of the pulse. Using Fig. 1(a) as a guide, we interpret our results as a spectral "fly-by" of a +1 STOV from the blue to the red side of our reference pulse spectrum centered at $800 \mathrm{~nm}$. We note that a similar fly-by of a -1 STOV from red to blue would present itself in an identical manner.

How are STOVs born in real collapsing pulses? In our $(r, z, \xi)$ simulations, vortices are immediately born as tori around the beam owing to azimuthally symmetric ( $\varphi$-independent $)$ phase shear. In real beam collapse, where there is $\varphi$ variation in the laser field, topological considerations lead us to expect that shear in higher $E$-field locations will first lead to a point null, followed by an expanding and distorted torus on one side of the beam that progressively wraps to the other side of the beam and then, meeting itself, splits into two toroidal STOVs of opposite phase winding. As might be expected from an electromagnetic field with spiral phase, STOVs possess angular momentum density, which we will discuss in a 
future publication. The onset of these STOVs, aligned with planes of constant $\xi$, has a beam-regularizing influence, as seen in the images of Fig. 5, which show remarkably flat phase and intensity profiles inside the ring. This could be the reason for the notably high-quality spatial modes and supercontinuum beams (so-called spatial cleaning [27]) observed in filamentation. We are performing 3D propagation simulations to verify this scenario. We also note that the ring null forms a natural and well-defined boundary between what had been qualitatively labeled the "core" and "reservoir" regions [14] in femtosecond filaments.

\section{STOV DYNAMICS AND ENERGY FLOW}

Once STOVs are generated, it is important to understand how they propagate. Following the method of Ref. [28] as applied to spatial vortices, we approximate the local form of the STOV as a spacetime " $R$-vortex," $\psi_{\text {vortex }} \equiv$ $\left(\xi-\xi_{0}\right) \pm i\left(r-r_{0}\right)$, of charge \pm 1 with a linear phase winding about $\left(\xi_{0}, r_{0}\right)$, embedded in a background field envelope $\psi_{\mathrm{bg}}$, such that $\psi=\psi_{\mathrm{bg}} \psi_{\text {vortex }}$. If we take $\psi_{\mathrm{bg}}=\rho e^{i \chi}$, where $\rho$ and $\chi$ are the real amplitude and phase of the background field, then as the pulse propagates along $z$, the nonlinear Schrödinger equation in $(r, z, \xi)$ moves the vortex location $\mathbf{r}_{\text {vortex }}=\left(r_{0}, \xi_{0}\right)$ according to

$$
\begin{aligned}
{\left[k \frac{\partial \mathbf{r}_{\text {vortex }}}{\partial z}=\right.} & \pm \frac{1}{\rho}\left(\hat{\mathbf{r}} \frac{\partial \rho}{\partial r}-\hat{\boldsymbol{\xi}} \beta_{2} \frac{\partial \rho}{\partial \xi}\right) \times \hat{\boldsymbol{\varphi}}+\left(\hat{\mathbf{r}} \frac{\partial \chi}{\partial r}-\hat{\boldsymbol{\xi}} \beta_{2} \frac{\partial \chi}{\partial \xi}\right) \\
& \left. \pm \hat{\boldsymbol{\xi}} \frac{1}{2 r}\right]\left.\right|_{(r, \xi, z)=\left(r_{0}, \xi_{0}, z_{0}\right)},
\end{aligned}
$$

where the derivatives are evaluated at the present vortex core location $\left(r_{0}, \xi_{0}, z_{0}\right)$ (see Appendix B). Equation (2) demonstrates interesting analogies with fluid vortices. The term $\pm \hat{\boldsymbol{\xi}} / 2 r$ propels the vortex forward or backward depending on its charge and radius (curvature), and strongly resembles the speed $\sim \Gamma / 4 \pi r$ of a toroidal fluid vortex (such as a smoke ring) [29]. Identifying $\hat{\mathbf{r}} \partial \chi / \partial r=\nabla_{\perp} \chi=k \mathbf{j}_{\perp} / \rho^{2}$ as the local effective velocity associated with the background electromagnetic flux (see Appendix C), we interpret it as a charge-independent draglike term, expanding (contracting) the STOV for power outflow (inflow) for $\partial \chi / \partial r>0(<0)$. The term $\pm \hat{\mathbf{r}} \times$ $\hat{\boldsymbol{\varphi}} \rho^{-1} \partial \rho / \partial r$ is a Magnus-like motion [1], here propelling the STOV along $\pm \hat{\xi}$, perpendicular to the vortex circulation vector $\hat{\boldsymbol{\varphi}}$ and the ring expansion or contraction direction $\hat{\mathbf{r}}$. The terms $-\hat{\boldsymbol{\xi}} \beta_{2} \partial \chi / \partial \xi$ and $\mp \hat{\boldsymbol{\xi}} \times \hat{\boldsymbol{\varphi}} \beta_{2} \rho^{-1} \partial \rho / \partial \xi$ are their spatiotemporal analogues. In gases, small $\beta_{2}\left(\sim 10^{-5}\right)$ makes these terms negligible; they contribute much more significantly in solid media.

We note that $\psi_{\mathrm{bg}}$ is not a fixed field independent of vortex motion. Equation (2) should be understood as a stepwise predictor of vortex motion based on an updated $\psi_{\mathrm{bg}}$.

To understand energy flow near STOVs, it is useful to consider the electromagnetic energy flux associated with the full field envelope $\psi=u e^{i \Phi}$ in the moving frame of the pulse (see Appendix C),

$$
\mathbf{j}=\frac{1}{k} u^{2}\left(\boldsymbol{\nabla}_{\perp} \Phi-\beta_{2} \frac{\partial \Phi}{\partial \xi} \hat{\xi}\right)
$$

where one can see that the sign of $\beta_{2}$ determines whether the energy flow near the STOV is spiral $\left(\beta_{2}<0\right)$ or saddle $\left(\beta_{2}>0\right)$. What are the relative contributions of longitudinal and transverse energy flow about a STOV? For filamentation in air, $\beta_{2} \sim 10^{-5}$, the characteristic axial pulse length and width are $\sim c \times 10$ fs and $\sim 100 \mu \mathrm{m}$ (filament core), and one finds $j_{\xi} / j_{r} \sim 10^{-4} \ll 1$. Here, the distinction between saddle and spiral is not important, as we are in the degenerate case. For solids, however, where $\beta_{2} \sim 10^{-2}$, we expect the distinction between saddle and spiral energy flow to be very important. Figure 6 provides intuitive visualization of the energy flow pattern for the saddle, degenerate, and spiral cases.

Equations (2) and (3) are useful for an intuitive picture of the governing dynamics of STOVs, especially when viewed
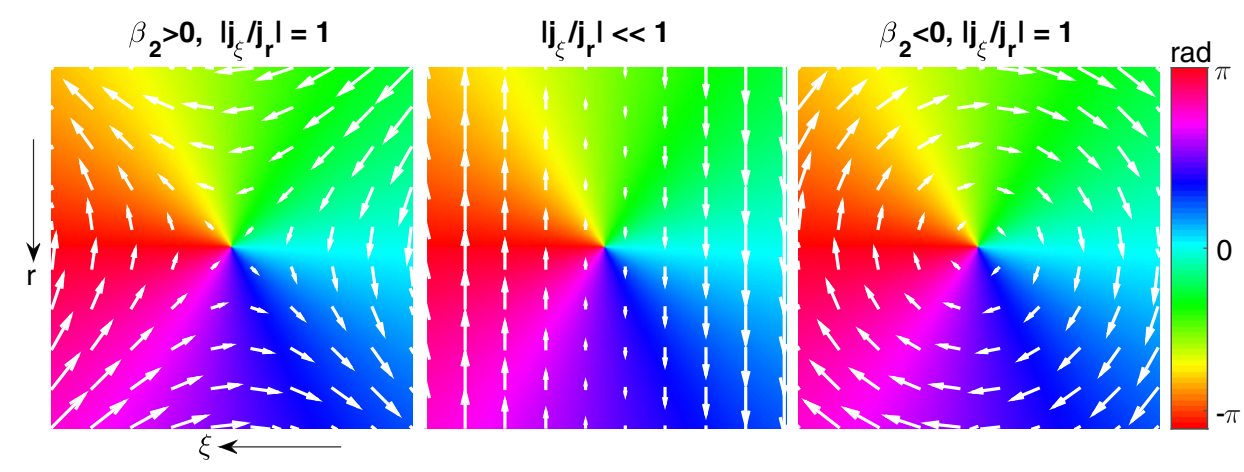

FIG. 6. Demonstration of energy flow about a +1 " $R$-vortex" STOV. White arrows correspond to size and direction of $\mathbf{j}$ in Eq. (3). There are three distinct regimes: the left-hand panel shows the saddle regime, which exists in regularly dispersive media $\left(\beta_{2}>0\right)$, the middle panel shows the degenerate regime, which can exist in regular or anomalously dispersive media and has a dominant axis for energy flow, and the right-hand panel shows the spiral regime, which exists in anomalously dispersive media $\left(\beta_{2}<0\right)$. 
together with propagation simulations. For example, for the four STOVs seen in the simulations of Fig. 1(a), their dominant early movement is governed by $\pm \hat{\boldsymbol{\xi}} \rho^{-1} \partial \rho / \partial r$ in Eq. (2), which propels the $+1(-1)$ STOV temporally forward (backward), with the forward motion initially being superluminal. (We will explore the detailed implications of superluminal STOV motion in a future publication.) A consequence of the opposing directions for the \pm charges is collision and annihilation of $v_{2}$ and $v_{3}$ of Fig. 1(a), as discussed earlier. Remarkably, the $v_{3}$ STOV superluminally climbs from a region of negligible intensity through many orders of magnitude of increasing intensity to reach and annihilate $v_{2}$, whereupon a local depression is left in the field that more gradually dissipates. The $v_{1}$ STOV eventually settles in the temporal middle of the highest intensity portion of the pulse, propagating at nearly $v_{g}$. Evidently, $\rho$ self-consistently evolves to balance the $\hat{\xi}$ terms in Eq. (2) and $\chi$ flattens along the radial dimension (as indicated in the experiment), preventing expansion or contraction of the STOV. Our simulations show that a surviving +1 STOV is always coupled to the filamenting pulse. This is no coincidence, as the energy flow for $\mathrm{a}+1$ STOV is toward (away from) the pulse axis temporally in front of (behind) the STOV. This is exactly as expected, where the front of the pulse draws energy in by Kerr selffocusing, and energy at the back of the pulse is directed outward by plasma refraction. A link and descriptions of STOV movies are in Ref. [30]. There appears to be a deep connection between the STOV picture of filamentationspontaneous generation of STOVs followed by STOVgoverned energy flow in the beam-and the spontaneous formation of conical nonlinear waves ( $\mathrm{X}$ and $\mathrm{O}$ waves), which have been used to explain propagation dynamics of a filamenting beam [31-33].

In real beams without $\varphi$ symmetry, we expect collisions of oppositely charged STOVs to be much more complex, although the beam regularization observed in experiments may conspire to promote collisions. Auxiliary 3D +1 linear propagation simulations, in which we impose STOVs as initial conditions on Gaussian beams, show repulsion of like-charged STOVs, which pass around each other, and splitting of higher charge STOVs into multiple STOVs of single charge. We note that our measured air-based STOVs are not solitons, as diffraction does not balance self-focusing for a dark object. STOV solitons could exist, however, in an anomalously dispersive, self-defocusing medium.

\section{CONCLUSIONS}

We have introduced the concept of the spatiotemporal optical vortex to ultrafast optics and demonstrated its existence. A STOV is an optical vortex with phase circulation in a spatiotemporal plane. STOVs form naturally as a consequence of arrested self-focusing collapse and their dynamics influences subsequent pulse propagation. STOVs can also be imposed linearly via prescribed spatiotemporal or spatiospectral phase shifts, making possible their engineering for applications. While evidence for STOV generation was demonstrated in experiments and simulations of short pulse filamentation in air, we expect that STOVs, whose dynamics are subject to topological constraints, are a fundamental and ubiquitous element of nonlinear propagation of intense pulses. STOV-STOV interactions should prove to be a fundamental mediator of intrabeam and interbeam dynamics.

\section{ACKNOWLEDGEMENTS}

The authors thank John Palastro (NRL) for the use of his propagation simulation (and permission to modify the source code), and Tony Ting (NRL), Dan Lathrop (UMd), and Peter Megson (UMd) for useful discussions. This work is supported by the Defense Advanced Research Projects Agency (Grant No. W911NF1410372), the Air Force Office of Scientific Research (Grants No. FA9550-13-10044 and FA9550-16-10121), the National Science Foundation (Grant No. PHY1301948), and the Army Research Office (Grant No. W911NF1410372).

\section{APPENDIX A: EXPERIMENTAL CONFIGURATION AND ANALYSIS}

\section{Experimental setup}

The experimental apparatus makes possible the reconstruction of the transverse spatial phase and intensity profiles of a femtosecond optical air filament in midflight. To do this, we use an abrupt air-helium transition to halt nonlinear propagation, as ionization yield and self-focusing are both negligible in helium. The beam is then relayed linearly from the transition zone through the helium and interfered with a reference pulse in an interferometer, enabling extraction of the transverse amplitude and phase profiles of the filament.

The experimental setup is shown in Fig. 7. Our filamentation source is a chirped pulse amplification Ti: sapphire amplifier $(\lambda=800 \mathrm{~nm}, 45 \mathrm{fs}, 0-5 \mathrm{~mJ})$. The beam from the laser is spatially filtered using a pinhole to produce a Gaussian mode with flat phase fronts - this is important for the reference arm in the experiment, which requires a flat spatial phase. After spatial filtering, the pumpfilamenting (signal) arm and reference arm are generated using uncoated wedges in a Mach-Zehnder (MZ) configuration to create a large difference in power between the two beams $\left(\sim 10^{4}: 1\right)$. Here, the low-power reference arm reflects off the front faces of the wedges, while the highpower signal arm transmits, creating a dispersion imbalance that is corrected further downstream. The beams are then compressed, with the signal arm now at 45-fs FWHM intensity, rotated $90 \mathrm{deg}$ using a periscope (converting $P$ polarization to $S$ ), and down collimated to a waist of $1.3 \mathrm{~mm}$ using a reflective off-axis telescope. 


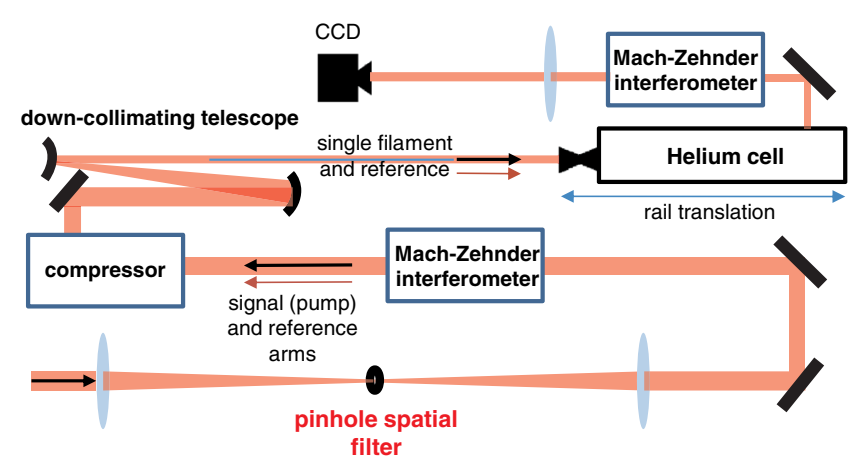

FIG. 7. Experimental setup for measuring the in-flight intensity and spatial phase profiles of collapsing and filamenting femtosecond pulses over a $\sim 2 \mathrm{~m}$ range.

After down collimation, the beams are launched a variable distance spanning 50-225 $\mathrm{cm}$ beyond the last optic of the telescope before nonlinear propagation terminates inside the nozzle of the translatable helium cell. Past the air-helium transition, both beams propagate linearly in helium $50 \mathrm{~cm}$, with the intense filament core of the high-power beam expanding transversely in size. Both beams are then wedge attenuated before being directed out of the cell through a $200-\mu \mathrm{m}$-thick BK7 window. Outside the cell, the high-power beam is attenuated to match the power of the reference arm via reflections from a second set of wedges in MZ configuration. In order to maintain polarization purity, polarization rotation from the upstream periscope is necessary, as wedge reflections preferentially select for $s$ polarization. Wedge transmissions by the reference arm fix the dispersion mismatch created in the precompressor MZ interferometer. The beams are recombined at the output of the interferometer and sent through a lens that images an upstream plane, just before the nozzle's gas transition region, to a CCD camera.

The air-helium interface is formed by the nonturbulent flow of helium, at slightly positive pressure, into the ambient air through a $1 / 4$ "-diameter nozzle on a translatable rail-mounted cell. The filament propagates from air into the nozzle and nonlinear propagation terminates over the sharp 4-mm transition from air to helium. The heliumair transition is measured, as in Ting et al. [26], by monitoring the strength of the $3^{3} \mathrm{D}-2^{3} \mathrm{P}, \lambda=587.6 \mathrm{~nm}$ helium line as the helium cell nozzle is moved through a tightly focused 800 -nm, 45-fs ionizing beam. As we show in Fig. 8(a), the rapid dropoff of the helium line indicates that there is negligible helium beyond a 4-mm 10\%-to-90\% transition layer at the nozzle.

To confirm the fidelity of imaging and phase reconstruction through the helium cell, we use a time domain propagation code [19] in $2+1$ dimensions $(r, z, \xi)$ to model the propagation of a filamenting beam through the 4-mm air-helium transition into the far field in the bulk helium at atmospheric pressure. The accuracy of phase and intensity reconstruction is verified by reverse propagating the beam via phase conjugation through vacuum back to
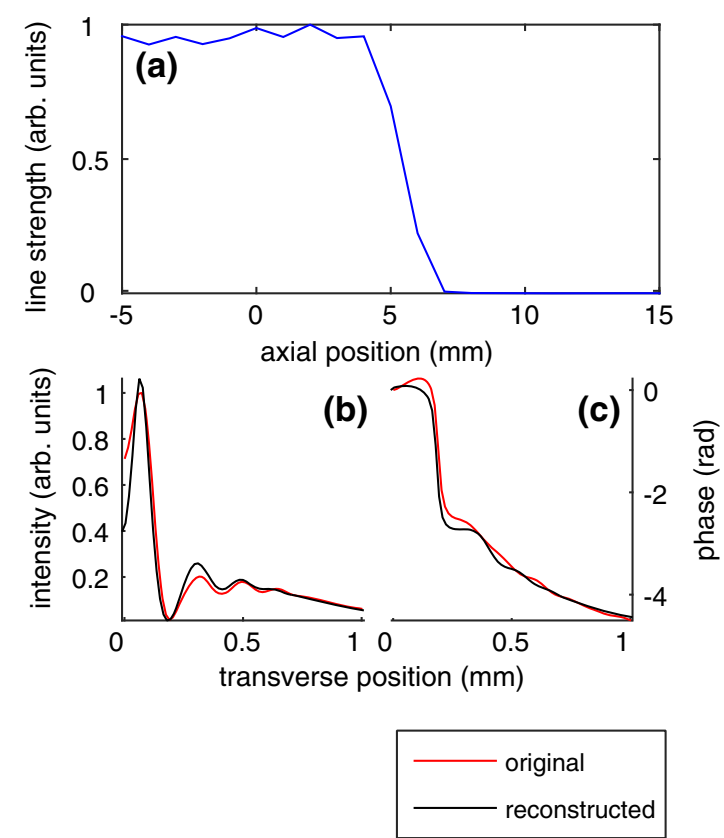

FIG. 8. (a) Line emission (in arbitrary units) of the neutral helium $3^{3} \mathrm{D}-2^{3} \mathrm{P}, \lambda=587.6 \mathrm{~nm}$ transition induced by a tightly focused $800 \mathrm{~nm}$ pulse as a function of helium cell position. (b) Simulated filament intensity and (c) spatial phase at $800 \mathrm{~nm}$ (black) just before the $\sim 4$-mm air-helium transition, as well as reconstructed intensity and phase (red). The reconstruction is performed by propagating the solution $4 \mathrm{~mm}$ through the transition region followed by $50 \mathrm{~cm}$ of helium. The simulated pulse is then backpropagated through $50.4 \mathrm{~cm}$ of vacuum to simulate reconstruction from imaging optics.

the air region just before the transition. The results are displayed in Figs. 8(b) and 8(c), which show that the reconstructed spatial intensity and phase at $800 \mathrm{~nm}$ (red) closely track that of the input electric field just before the transition region (black). In addition, we verify that small deviations from the correct imaging plane $( \pm 1 \mathrm{~cm})$ do not affect the results.

\section{STOV in spatiospectral space}

Although the STOV is a vortex in the spatiotemporal domain, our experiment measures the spatial phase of the filamenting pulse by interference with a reference pulse that is centered at $\lambda=800 \mathrm{~nm}$ (see Appendix A 3). What is the signature of a STOV in spatiospectral space?

Consider an " $R$ " vortex ring centered at $\left(r_{0}, \xi_{0}\right)$ embedded in a Gaussian background field with a temporal chirp,

$E(r, \xi)=E_{0} e^{-r^{2} / w_{r}^{2}-\xi^{2} / w_{\xi}^{2}} e^{-i a \xi^{2}}\left[\xi-\xi_{0} \pm i\left(r-r_{0}\right)\right]$,

where $w_{r}$ and $w_{\xi}$ are transverse and longitudinal widths, respectively, and $a$ is a chirp parameter. The Fourier transform along the $\xi$ axis is, up to a complex coefficient, 


$$
\begin{aligned}
\tilde{E}(r, \omega) \propto & e^{-r^{2} / w_{r}^{2}-\left(\omega-\omega_{c}\right)^{2} /\left[v_{g}^{2}\left(i a-w_{\xi}^{-2}\right)\right]} \\
& \times\left\{\left(2+2 i a w_{\xi}^{2}\right) \xi_{0}-\frac{i w_{\xi}^{2}\left(\omega-\omega_{c}\right)}{v_{g}}\right. \\
& \left. \pm 2\left(r-r_{0}\right)\left(a w_{\xi}^{2}-i\right)\right\}
\end{aligned}
$$

where $\omega \equiv k_{\xi} v_{g}$, the variable conjugate to $\xi$ is $k_{\xi}$, and $\omega_{c}$ is the central frequency of the pulse. In spatiospectral space the vortex is centered at

$$
\begin{aligned}
r_{0}^{\prime} & =r_{0}\left(1 \mp \frac{\xi_{0}}{r_{0}} \frac{1}{a w_{\xi}^{2}}\right), \\
\omega_{0} & =2 \xi_{0} v_{g}\left(\frac{1}{a w_{\xi}^{4}}+a\right)+\omega_{c} .
\end{aligned}
$$

It is seen that the STOV is manifested at a definite spectral location $\omega_{0}$ depending on its axial position $\xi_{0}$ and pulse chirp $a$. For a STOV perfectly centered in the pulse $\left(\xi_{0}=0\right)$, its spatiospectral representation is located at the central frequency $\left(\omega=\omega_{c}\right)$ and at the same spatial radius $\left(r_{0}^{\prime}=r_{0}\right)$. For an air filament, the $\mathrm{STOV}$ is, indeed, positioned temporally about the peak intensity of the filamenting pulse, as this is where the Kerr effect-driven inward flow of optical energy yields to the plasma refraction-induced outflow.

Figure 9 displays the spatiospectral representation of a STOV using simulation output. We use the same simulation parameters presented in Fig. 3: $w_{0}=1.3 \mathrm{~mm}$, pulse energy $=2.8 \mathrm{~mJ}\left(P / P_{\mathrm{cr}}=6.4\right)$, and pulse FWHM $45 \mathrm{fs}$. The top row shows the spatiospectral phase, while the bottom row displays the spatiospectral intensity. The leftmost column shows the complete spatiospectral phase and intensity, and makes clear that there is phase vorticity in the spatiospectral domain. The middle (rightmost) column shows the phase and intensity spectrally fore (aft) of the vortex, where we present the data in a manner similar to the experimental images of Fig. 5. Here, "fore" and "aft" refer to left and right of the dashed black line in the upper lefthand panel. The diameter of the core is well reproduced, as are the abrupt phase jumps of $\sim \pm \pi$ from core to periphery on both sides of the vortex. The spectral intensity images show the dark ring of the vortex core. The location of the vortex core changes as the beam propagates. The phase flip seen in Fig. 9 by sampling spectrally to either side of the core would also be seen by observing a fixed frequency while scanning the input power or by scanning along the propagation axis of the filament, which is how we observe the phase flip experimentally in Figs. 4 and 5.
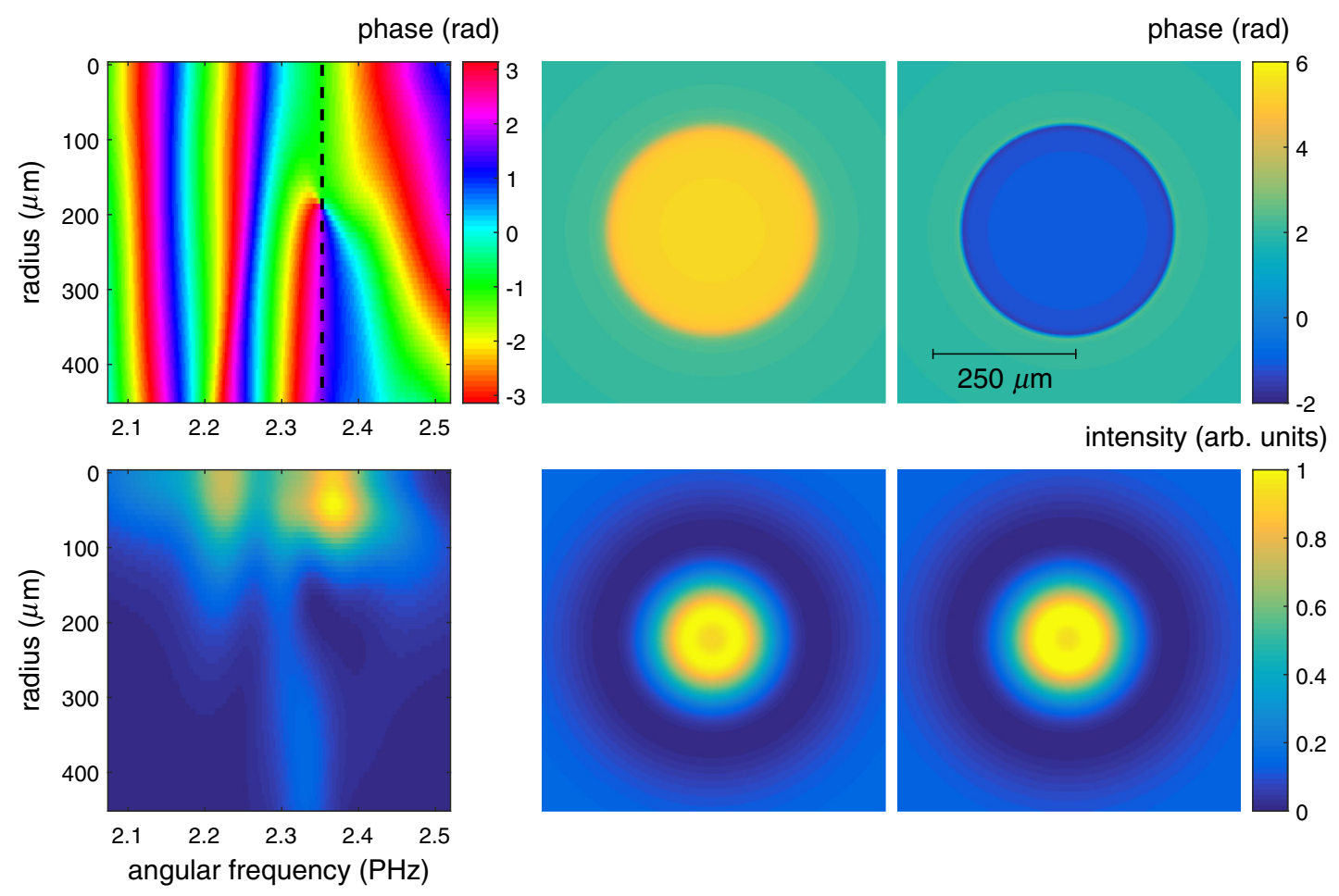

FIG. 9. Spectral phase (radians, top) and spectral intensity (arbitrary units, bottom) taken from simulation at $z=180 \mathrm{~cm}$ as the vortex core in the spatiospectral domain crosses $2.35 \mathrm{PHz}(\lambda=800 \mathrm{~nm})$. Simulation parameters are the same as Fig. 3 of the main text: $w_{0}=1.3 \mathrm{~mm}$, pulse energy $2.8 \mathrm{~mJ}\left(P / P_{\mathrm{cr}}=6.4\right)$, pulse FWHM $45 \mathrm{fs}$. From left to right, the top row (bottom row) shows the full spatial-spectral phase (intensity), as well as spatial phase (intensity) slices spectrally fore and aft of $800 \mathrm{~nm}$. The dashed black line in the top left-hand panel intersects the vortex core. 


\section{Interferometric reconstruction}

Since the collimated low-power reference arm has a flat spatial phase, the spatial phase difference, extracted from the interferogram using standard techniques [34,35], is just the spatial phase accumulated by the nonlinearly propagating beam. Such a pulse can develop a complicated time (and, therefore, frequency) dependence [14,36,37]. However, our interferograms are spectrally gated by the narrower reference pulse spectrum. What is actually measured, therefore, is a weighted average of the spatial phase as a function of frequency, where the weighting factor is the product of the (narrower) spectral amplitude of the lowpower reference arm and the (broader) spectral amplitude of the filamenting arm. The oscillatory portion of the signal on the $\mathrm{CCD}$ is given by

$\operatorname{int}(x, y)=2 \operatorname{Re}\left[e^{-i k x \sin \theta} \int_{-\infty}^{\infty} d \omega A_{\mathrm{ref}}^{*}(x, y, \omega) A_{\mathrm{sig}}(x, y, \omega)\right]$,

where $x$ and $y$ are transverse coordinates in the beam, $A_{\text {ref }}$ and $A_{\text {sig }}$ are Fourier transforms of the field envelopes of the reference and signal pulses, $k$ is the central wave number of the reference pulse, and $\theta$ is the crossing angle of the two beams. As the spatially filtered reference pulse propagates linearly, $A_{\text {ref }}$ is fully known, and is well approximated by a Gaussian with flat spectral phase, $A_{\text {ref }}(x, y, \omega)=A_{0} e^{-\left(x^{2}+y^{2}\right) / w^{2}-\omega^{2} / \omega_{0}^{2}}$, and the weighted spatiospectral phase of the signal beam is then extracted.

What is the difference between the weighted spatial phase given in Eq. (A4) and the spatial phase at a single frequency

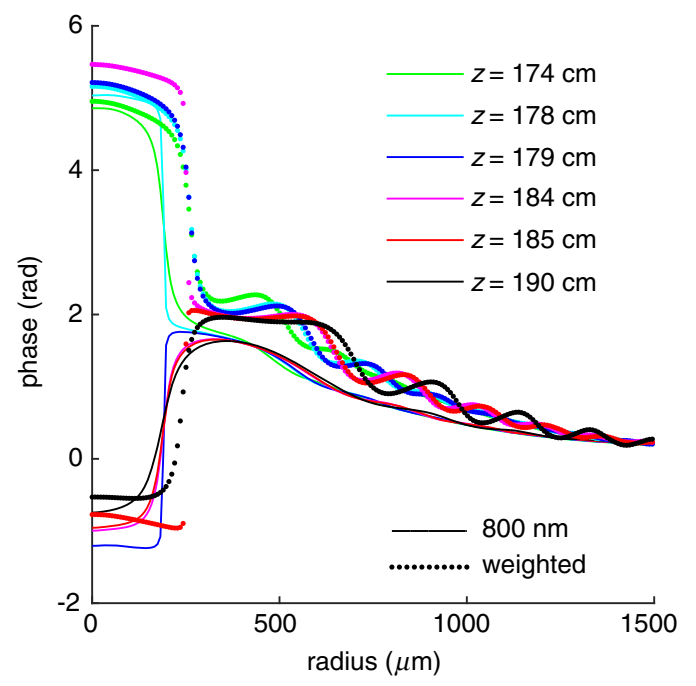

FIG. 10. Comparison of the spatial-spectral phase of the beam at $800 \mathrm{~nm}$ to the weighted spatial phase measured about $800 \mathrm{~nm}$ using the same simulation parameters considered in Fig. 3: $w_{0}=1.3 \mathrm{~mm}$, pulse energy $2.8 \mathrm{~mJ}\left(P / P_{\mathrm{cr}}=6.4\right)$, pulse FWHM 45 fs. Flipping of the spatial phase occurs at $z=179 \mathrm{~cm}$ at $800 \mathrm{~nm}$ and $z=185 \mathrm{~cm}$ for the weighted spatial phase. (such as at the spectral center $\lambda=800 \mathrm{~nm}$ ), as discussed in Appendix A 2? Figure 10 uses simulation output to directly compare the spatiospectral phase at $\lambda=800 \mathrm{~nm}$ and the weighted spatiospectral phase. We simulate using the same input parameters as Fig. 3: $w_{0}=1.3 \mathrm{~mm}$, pulse energy $2.8 \mathrm{~mJ}\left(P / P_{\mathrm{cr}}=6.4\right)$, pulse FWHM 45 fs. The figure tracks the evolution of the two different spatial phase quantities as the STOV in spatiospectral space crosses $\lambda=800 \mathrm{~nm}$. It is apparent from Fig. 10 that the two quantities follow each other closely, and, critically, they both exhibit the flip in phase we use to identify the vortex. The two main differences in the phase quantities are that the $800-\mathrm{nm}$ spatial phase flips first at $z=179 \mathrm{~cm}$, followed by the weighted phase at $z=185 \mathrm{~cm}$ (likely due to asymmetric dispersion about $800 \mathrm{~nm}$ ), and that the weighted phase gives a core region $\sim 20 \%$ larger than the exact spatial phase at $800 \mathrm{~nm}$. Figure 10 establishes that the weighted spatial phase is a good proxy for the spatial phase at $\lambda=800 \mathrm{~nm}$ and can be used to detect the STOV in spatiospectral space as outlined in Appendix A 2.

\section{APPENDIX B: SPATIOTEMPORAL OPTICAL VORTEX EQUATIONS OF MOTION}

We assume azimuthal symmetry and consider the evolution of the complex envelope $\psi$ associated with the scalar electric field $E$,

$$
E(r, \xi, z)=\psi(r, \xi, z) e^{i(k z-\omega t)},
$$

where $k=2 \pi / \lambda, \xi=v_{g} t-z$, and $v_{g}$ is the group velocity. In the paraxial and slowly varying envelope approximation, the propagation equation for $\psi$ is

$$
2 i k \frac{\partial \psi}{\partial z}+\nabla_{\perp}^{2} \psi-\beta_{2} \frac{\partial^{2} \psi}{\partial \xi^{2}}+k^{2} V(\psi) \psi=0,
$$

where $\nabla_{\perp}^{2}=\partial^{2} / \partial r^{2}+(1 / r)(\partial / \partial r), \beta_{2}=c^{2} k_{0}\left(\partial^{2} k / \partial \omega^{2}\right)_{0}$ is the dimensionless group velocity dispersion, and $V(\psi)$ is a nonlinear term. For the case $\beta_{2}=0$, Eq. (B2) drives transverse displacement of purely spatial optical vortices through the term $\nabla_{\perp}^{2} \psi$, as shown in Refs. [28,38]. Here, the term containing $\beta_{2}$ additionally drives vortex motion in the local space $(\xi)$ direction. Therefore, as the beam propagates, the vortex moves temporally as well.

Suppose that at $z_{0}$ the position of the vortex ring is $\mathbf{r}_{\mathrm{vortex}}=\left(\xi_{0}, r_{0}\right)$, and at $z_{0}+d z$ the position of the vortex ring is $\mathbf{r}_{\text {vortex }}+d \mathbf{r}_{\text {vortex }}=\left(\xi_{0}+d \xi, r_{0}+d r\right)$. Then, $\psi\left(r_{0}+\right.$ $\left.d r, \xi_{0}+d \xi, z_{0}+d z\right) \approx \psi\left(r_{0}, \xi_{0}, z_{0}\right)+(\partial \psi / \partial r) d r+(\partial \psi /$ $\partial \xi) d \xi+(\partial \psi / \partial z) d z$. But since $\psi=0$ at the vortex, this leads to

$$
\boldsymbol{\nabla}_{\mathrm{ST}} \psi \cdot d \mathbf{r}_{\mathrm{vortex}}=-\frac{\partial \psi}{\partial z} d z
$$


where the spacetime gradient is defined as $\boldsymbol{\nabla}_{\mathrm{ST}}=$ $(\partial / \partial r) \hat{\mathbf{r}}+(\partial / \partial \xi) \hat{\boldsymbol{\xi}}$.

Following the method of Ref. [28] as applied to spatial vortices, we approximate the local form of the STOV as a spacetime $R$-vortex, $\psi_{\text {vortex }} \equiv\left(\xi-\xi_{0}\right) \pm i\left(r-r_{0}\right)$, of charge \pm 1 with a linear phase winding about $\left(\xi_{0}, r_{0}\right)$, embedded in a background field envelope $\psi_{\text {bg }}$ such that $\psi=\psi_{\text {bg }} \psi_{\text {vortex }}$. If we take $\psi_{\text {bg }}=\rho e^{i \chi}$, where $\rho$ and $\chi$ are the real amplitude and phase of the background field, then substitution of $\psi=\psi_{\mathrm{bg}} \psi_{\text {vortex }}$ into Eq. (B2) yields

$$
\begin{aligned}
k \frac{\partial \mathbf{r}_{\mathrm{vortex}}}{\partial z}= & \pm \frac{1}{\rho}\left(\hat{\mathbf{r}} \frac{\partial \rho}{\partial r}-\hat{\boldsymbol{\xi}} \beta_{2} \frac{\partial \rho}{\partial \xi}\right) \\
& \times \hat{\boldsymbol{\phi}}\left(\hat{\mathbf{r}} \frac{\partial \chi}{\partial r}-\hat{\boldsymbol{\xi}} \beta_{2} \frac{\partial \chi}{\partial \xi}\right) \pm \hat{\boldsymbol{\xi}} \frac{1}{2 r}
\end{aligned}
$$

This is Eq. (2) in the main text. As the pulse propagates along $z$, Eq. (B4) gives the next move of the STOV based on the current self-consistent background field $\psi_{\mathrm{bg}}$.

\section{APPENDIX C: ENERGY CURRENT DENSITY}

Without loss of generality, we take $\psi=u e^{i \Phi}$, where $u$ and $\Phi$ are the real amplitude and phase of the field. Based on conservation of energy, we can derive an equation of the form $(\partial / \partial z) u^{2}=-\boldsymbol{\nabla} \cdot \mathbf{j}$, where $\mathbf{j}$ is the energy current density in the laser pulse frame and $u^{2}=|\psi|^{2}$ is the normalized energy density. Following an argument similar to Ref. [39], in order to derive $\mathbf{j}$ we start with $(\partial / \partial z) u^{2}=$ $(\partial / \partial z)\left(\psi^{*} \psi\right)=\psi^{*}(\partial / \partial z) \psi+$ c.c. and replace $(\partial / \partial z) \psi$ using Eq. (B2). Near the vortex, the amplitude of the field is close to zero, so loss mechanisms like ionization and molecular rotations can be neglected, resulting in $\psi^{*}(1 / 2 i k) V \psi+$ c.c. $=0 \quad(V\{\psi\} \quad$ is a real quantity $)$. Furthermore, for other terms, after canceling out the purely imaginary terms with their complex conjugates, we obtain $(-1 / 2 i k) \psi^{*} \nabla_{\perp}^{2} \psi+$ c.c $=-(1 / k)\left(u^{2} \nabla_{\perp}^{2} \Phi+\nabla_{\perp} u^{2} \cdot \nabla_{\perp} \Phi\right)$ and $\left(\beta_{2} / 2 i k\right) \psi^{*}\left(\partial^{2} / \partial \xi^{2}\right) \psi+$ c.c. $=\left(\beta_{2} / k\right)\left[u^{2}\left(\partial^{2} / \partial \xi^{2}\right) \Phi+\right.$ $\left.(\partial / \partial \xi) u^{2}(\partial / \partial \xi) \Phi\right]$, resulting in an energy current density of

$$
\mathbf{j}=\frac{1}{k} u^{2}\left(\boldsymbol{\nabla}_{\perp} \Phi-\beta_{2} \frac{\partial \Phi}{\partial \xi} \hat{\xi}\right)
$$

[1] K. Batchelor, An Introduction to Fluid Dynamics (Cambridge University Press, Cambridge, England, 1977).

[2] M. J. H. Ku, W. Ji, B. Mukherjee, E. Guardado-Sanchez, L. W. Cheuk, T. Yefsah, and M. W. Zwierlein, Motion of a Solitonic Vortex in the BEC-BCS Crossover, Phys. Rev. Lett. 113, 065301 (2014).
[3] S. Donadello, S. Serafini, M. Tylutki, L. P. Pitaevskii, F. Dalfovo, G. Lamporesi, and G. Ferrari, Observation of Solitonic Vortices in Bose-Einstein Condensates, Phys. Rev. Lett. 113, 065302 (2014).

[4] G. P. Bewley, D. P. Lathrop, and K. R. Sreenivasan, Superfluid Helium: Visualization of Quantized Vortices, Nature (London) 441, 588 (2006).

[5] M. R. Dennis, Y.S. Kivshar, M. S. Soskin, and G. A. Swartzlander, Jr., Singular Optics: More Ado About Nothing, J. Opt. A 11, 090201 (2009).

[6] J. F. Nye and M. V. Berry, Dislocations in Wave Trains, Proc. R. Soc. A 336, 165 (1974).

[7] L. Allen, M. W. Beijersbergen, R. J. C. Spreeuw, and J. P. Woerdman, Orbital Angular Momentum of Light and the Transformation of Laguerre-Gaussian Laser Modes, Phys. Rev. A 45, 8185 (1992).

[8] M. W. Beijersbergen, R. P. C. Coerwinkel, M. Kristensen, and J. P. Woerdman, Helical-Wavefront Laser Beams Produced with a Spiral Phaseplate, Opt. Commun. 112, 321 (1994).

[9] F. Eilenberger, K. Prater, S. Minardi, R. Geiss, U. Röpke, J. Kobelke, K. Schuster, H. Bartelt, S. Nolte, A. Tünnermann, and T. Pertsch, Observation of Discrete, Vortex Light Bullets, Phys. Rev. X 3, 041031 (2013).

[10] D. Mihalache, D. Mazilu, F. Lederer, Y. V. Kartashov, L.-C. Crasovan, L. Torner, and B. A. Malomed, Stable Vortex Tori in the Three-Dimensional Cubic-Quintic Ginzburg-Landau Equation, Phys. Rev. Lett. 97, 073904 (2006).

[11] K. W. Nicholls and J. F. Nye, The Paths of Dislocations in Wave Pulses: An Experimental Test, J. Phys. A 19, 375 (1986).

[12] Y. V. Kartashov, B. A. Malomed, Y. Shnir, and L. Torner, Twisted Toroidal Vortex Solitons in Inhomogeneous Media with Repulsive Nonlinearity, Phys. Rev. Lett. 113, 264101 (2014).

[13] G. Fibich, The Nonlinear Schrödinger Equation: Singular Solutions and Optical Collapse (Springer, New York, 2015).

[14] A. Couairon and A. Mysyrowicz, Femtosecond Filamentation in Transparent Media, Phys. Rep. 441, 47 (2007).

[15] D. Strickland and P. B. Corkum, Resistance of Short Pulses to Self-Focusing, J. Opt. Soc. Am. B 11, 492 (1994).

[16] P. Panagiotopoulos, P. Whalen, M. Kolesik, and J. V. Moloney, Super High Power Mid-Infrared Femtosecond Light Bullet, Nat. Photonics 9, 543 (2015).

[17] G. Fibich and B. Ilan, Vectorial and Random Effects in Self-Focusing and in Multiple Filamentation, Physica (Amsterdam) 157D, 112 (2001).

[18] A. J. Goers, G. A. Hine, L. Feder, B. Miao, F. Salehi, J. K. Wahlstrand, and H. M. Milchberg, Multi-MeV Electron Acceleration by Subterawatt Laser Pulses, Phys. Rev. Lett. 115, 194802 (2015).

[19] J. P. Palastro, T. M. Antonsen, Jr., and H. M. Milchberg, Compression, Spectral Broadening, and Collimation in Multiple, Femtosecond Pulse Filamentation in Atmosphere, Phys. Rev. A 86, 033834 (2012).

[20] M. Kolesik and J. V. Moloney, Nonlinear Optical Pulse Propagation Simulation: From Maxwell's to Unidirectional Equations, Phys. Rev. E 70, 036604 (2004); J. Andreasen and M. Kolesik, Nonlinear Propagation of Light in Structured Media: Generalized Unidirectional Pulse Propagation Equations, Phys. Rev. E 86, 036706 (2012). 
[21] E. W. Rosenthal, J. P. Palastro, N. Jhajj, S. Zahedpour, J. K. Wahlstrand, and H. M. Milchberg, Sensitivity of Propagation and Energy Deposition in Femtosecond Filamentation to the Nonlinear Refractive Index, J. Phys. B 48, 094011 (2015).

[22] J. K. Wahlstrand, Y.-H. Cheng, and H. M. Milchberg, Absolute Measurement of the Transient Optical Nonlinearity in $\mathrm{N}_{2}, \mathrm{O}_{2}, \mathrm{~N}_{2} \mathrm{O}$, and Ar, Phys. Rev. A 85, 043820 (2012).

[23] A. S. Desyatnikov, D. Buccoliero, M. R. Dennis, and Y. S. Kivshar, Spontaneous Knotting of Self-Trapped Waves, Sci. Rep. 2, 771 (2012).

[24] K. D. Moll, A. L. Gaeta, and G. Fibich, Self-Similar Optical Wave Collapse: Observation of the Townes Profile, Phys. Rev. Lett. 90, 203902 (2003).

[25] J. K. Wahlstrand, Y.-H. Cheng, and H. M. Milchberg, High Field Optical Nonlinearity and the Kramers-Kronig Relations, Phys. Rev. Lett. 109, 113904 (2012).

[26] A. Ting, D. F. Gordon, E. Briscoe, J. R. Penano, and P. Sprangle, Direct Characterization of Self-Guided Femtosecond Laser Filaments in Air, Appl. Opt. 44, 1474 (2005).

[27] B. Prade, M. Franco, A. Mysyrowicz, A. Couairon, H. Buersing, B. Eberle, M. Krenz, D. Seiffer, and O. Vasseur, Spatial Mode Cleaning by Femtosecond Filamentation in Air, Opt. Lett. 31, 2601 (2006).

[28] D. Rozas, C. T. Law, and G. A. Swartzlander, Jr., Propagation Dynamics of Optical Vortices, J. Opt. Soc. Am. B 14, 3054 (1997).

[29] I. S. Sullivan, J. J. Niemela, R. E. Hershberger, D. Bolster, and R. J. Donnelly, Dynamics of Thin Vortex Rings, J. Fluid Mech. 609, 319 (2008).

[30] See STOV movies at http://lasermatter.umd.edu. Movie captioned "STOV birth and collision": Initial pulse is collimated with Gaussian beam waist $w_{0}=1.3 \mathrm{~mm}$, 45 fs FWHM intensity beam, and energy $2.8 \mathrm{~mJ}$. The beam is propagating in air. From $z=130 \mathrm{~cm}$ to $z=160 \mathrm{~cm}$, the beam undergoes optical collapse, and then generates 4 STOVs from $z=163 \mathrm{~cm}$ to $z=167 \mathrm{~cm}$. There is a STOV annihilation event at $z=204 \mathrm{~cm}$. Movie captioned "STOV settles with main filamenting pulse": Same simulation input parameters as "STOV birth and collision," but with window zoomed in on the temporally foremost filamenting pulse.
We see the +1 STOV, which forms with the pulse at optical collapse $(z=167 \mathrm{~cm})$, settle around the foremost pulse. There is an additional vortex pair that forms at $z=219 \mathrm{~cm}$. The +1 STOV subsequently annihilates on axis at $z=232 \mathrm{~cm}$, while the -1 STOV is annihilated by another vortex that forms on axis at $z=243 \mathrm{~cm}$ and is then almost immediately annihilated by another collision at $z=244 \mathrm{~cm}$.

[31] M. Kolesik, E. W. Wright, and J. V. Moloney, Dynamic Nonlinear X Waves for Femtosecond Pulse Propagation in Water, Phys. Rev. Lett. 92, 253901 (2004).

[32] D. Faccio, A. Matijosius, A. Dubietis, R. Piskarskas, A. Varanavicius, E. Gaizauskas, A. Piskarskas, A. Couairon, and P. Di Trapani, Near- and Far-Field Evolution of Laser Pulse Filaments in Kerr Media, Phys. Rev. E 72, 037601 (2005).

[33] D. Faccio, A. Averchi, A. Lotti, P. Di Trapani, A. Couairon, D. Papazoglou, and S. Tzortzakis, Ultrashort Laser Pulse Filamentation from Spontaneous X Wave Formation in Air, Opt. Express 16, 1565 (2008).

[34] M. Takeda, H. Ina, and S. Kobayashi, Fourier Transform Method of Fringe-Pattern Analysis for Computer-Based Topography and Interferometry, J. Opt. Soc. Am. 72, 156 (1982).

[35] T. R. Clark and H. M. Milchberg, Time- and Space-Resolved Density Evolution of the Plasma Waveguide, Phys. Rev. Lett. 78, 2373 (1997).

[36] A. Couairon, J. Biegert, C. P. Hauri, W. Kornelis, F. W. Helbing, U. Keller, and A. Mysyrowicz, Self-Compression of Ultra-Short Laser Pulses Down to One Optical Cycle by Filamentation, J. Mod. Opt. 53, 75 (2006).

[37] S. Skupin, G. Stibenz, L. Bergé, F. Lederer, T. Sokollik, M. Schnürer, N. Zhavoronkov, and G. Steinmeyer, SelfCompression by Femtosecond Pulse Filamentation: Experiments versus Numerical Simulations, Phys. Rev. E 74, 056604 (2006).

[38] G. Indebetouw, Optical Vortices and Their Propagation, J. Mod. Opt. 40, 73 (1993).

[39] D. Faccio, A. Lotti, A. Matijosius, F. Bragheri, V. Degiorgio, A. Couairon, and P. Di Trapani, Experimental Energy-Density Flux Characterization of Ultrashort Laser Pulse Filaments, Opt. Express 17, 8193 (2009). 